\title{
First microRNA mimic enters clinic
}

In April, Austin, Texas-based Mirna Therapeutics began dosing patients with MRX34, the first microRNA (miRNA) mimic to reach phase 1 studies. Other companies in this emerging field greeted the news with excitement, yet cautioned that potential off-target side effects and dosage issues could arise from this entirely novel approach to treating disease. miRNAs are short (20-25 nucleotides), occur naturally in the cell and help regulate gene expression by interacting with complementary mRNAs. Because each one modulates tens to hundreds of genes, miRNAs simultaneously control multiple cellular pathways, and when deregulated, contribute to disease.

In cancer, miRNAs are frequently overexpressed or downregulated, and companies are developing therapeutics to correct aberrant expression. With MRX34, which is entering a phase 1 trial in patients with primary liver cancer or metastatic cancer with liver involvement, Mirna's strategy is to restore lost suppressor function of endogenous miR-34 using a synthetic miRNA mimic. The therapeutic MRX34 directly regulates at least 24 known oncogenes, such as those involved in the cell cycle and proliferation, anti-apoptosis, metastasis, chemoresistance, cancer cell self-renewal and oncogenic transcription. "People have been focused on targeted therapies for many years now, but tumors often find a way to get around the blocks these

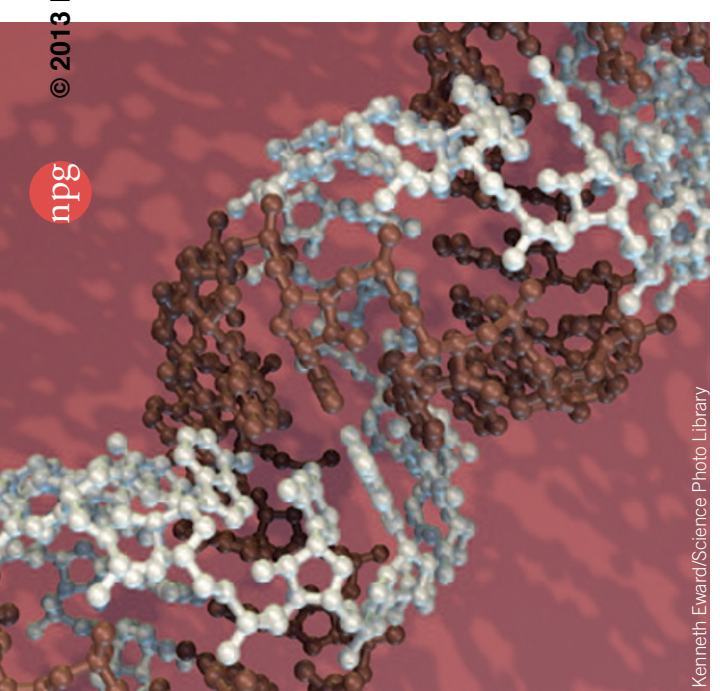

miRNA uses double-stranded RNA delivered by liposomes that naturally accumulate in the liver.

drugs put up," explains Mirna president and CEO Paul Lammers. "So attacking more of those pathways all at once might be a great new way to go after tumors."

MRX34 is a double-stranded RNA delivered by Smarticles, a liposome technology licensed from Marina Biotech of Bothell, Washington. Liposomes naturally accumulate in the liver, making liver cancer a logical first indication for MRX34. Because Smarticles (which comprise different mixtures of palmitoyl oleoyl phosphatidyl choline, dioleoyloxytrimethylammonium propane, 1,2-dimyristoylglycerol3-hemisuccinate and cholesterol) are anionic at normal body $\mathrm{pH}$ but cationic at lower $\mathrm{pH}$, uptake into tumors-which tend to have lower $\mathrm{pH}$-is enhanced and unwanted interactions with healthy cells prevented, a finding that was borne out in preclinical studies. MRX34 restores the tumor suppressor pathway to normal, inducing apoptosis in tumor cells in vitro and in mouse models of cancer. Studies also show that at the anticipated therapeutic doses, no changes in cytokine profiles are apparent. But others in the field have raised concerns about Mirna's "replacement" strategy. William Marshall, president and CEO of Boulder, Colorado-based miRagen's Therapeutics, noted that introducing miRNA into a cell type that wouldn't normally express that miRNA could result in off-target side effects. "With a microRNA replacement therapy, I would want a microRNA that is broadly expressed in many tissues and is downregulated only in diseased cells. I think that is true for miR-34," says Marshall. Lammers says he is unaware of any research showing that miR34 is absent in any type of cell in the body.

Another general safety concern with miRNA mimics is dosing. "We don't yet fully understand the pharmacokinetics of microRNA, that is, we don't know exactly what will happen when you increase cellular levels by 10 -fold versus 100 -fold versus 1,000-fold," according to Serge Patrick Nana-Sinkam, a practicing pulmonologist and associate professor of internal medicine at The James Comprehensive Cancer Center of The Ohio State University in Columbus. He adds, "A higher dosage level might actually cause an opposite effect."

Mirna will not know the magnitude of increase in miR-34 cellular levels required for efficacy in humans until biopsy material from patients in clinical trials becomes available. Lammers says healthy liver cells normally have about 300 copies of miR-34, whereas there are only about 100 copies in a liver cancer cell. "I don't think we have to bring it back to 300 copies per cell because there seems to be a threshold system at work here above which the tumor suppression system will turn back on," he says, adding that having access to and performing analyses of tumor biopsies could help determine if such a threshold phenomenon truly is in play.

Aaron Bouchie Ithaca, NY 